\title{
De l'eau pour tous ?
}

\author{
Water for everybody?
}

par T. Chambolle

Suez Lyonnaise des Eaux

The experience that Lyonnaise des Eaux has acquired in its contracts in Argentina has shown up the problem of serving districts with low financial resources, under the general conditions of a contract which does not take into account the capacity to pay of the poorest populations.

As this problem is also encountered in the contracts of La Paz, Manila, Jakarta, Casablanca etc., it was urgent to undertake a reflection on the subject. A project was financed in 1997.

Before tackling the heart of the subject, it is useful to recall some basic ideas forming the basis of the reflection.

One can observe a substantial increase in the number of cities with populations of more than a million: 157 in 1975 , 320 in 2000. It is estimated that a large part of the people without access to drinking water or sanitation (1000 million and 2900 million respectively) live in underprivileged urban environments.

In underprivileged districts, the technical impossibility (no roads or even any trace of them), the institutional impossibility (occupation of the land which is often illegal) and the financial impossibility (cost of a normal type of supply by a network with connections, relative to the population's capacity to pay) of providing in the short term a conventional water supply and sanitation service to the populations concerned constitute a considerable problem.

The report proposes an approach wich best allows the situation of underpriviliged districts to be dealt with. This approach may be summarized as follows :

- Development of appropriate contracts,

- Integration of the problem of underprivileged districts into the Master Plan as far upstream as possible,

- Implementation of transitory and progressive solutions.

\section{I 口 INTRODUCTION}

L'expérience que Suez Lyonnaise des Eaux a acquise dans ses contrats argentins a mis en évidence la problématique de desservir des quartiers à faibles ressources financières, à partir de conditions générales fixées au contrat ne tenant pas compte de la capacité à payer des populations les plus démunies.

Comme cette problématique devait se retrouver sur les contrats de La Paz, Manille, Djakarta, Casablanca, etc., il était indispensable de mener une recherche sur ce sujet. Un projet a été financé par le fonds RDI (Recherche, Développement, Innovation) et le PRAME (Programme de Recherche Appliquée aux Métiers de l'Eau) en 1997. Il visait essentiellement l'accompagnement des projets argentins, le recensement des expériences dans le groupe et hors groupe, ainsi que le recensement des solutions apportées.

Ce projet a été piloté par la Direction Technique et de la Recherche (Lisette Provencher), en collaboration avec SAFEGE, et mené à bien grâce à la coopération de nom- breuses personnes, provenant de diverses entités dans le groupe : Aguas Argentinas, Aguas Provinciales de Santa $\mathrm{Fe}$, Aguas del Illimani (La Paz), Water and Sanitation Services (WSSA, Afrique du Sud), le CIRSEE, le NLTRC (Northumbrian Lyonnaise Technology Research Centre), Aquassistance, la Direction Internationale des Projets, la Direction Clientèle et EDA.

\section{QUELQUES RÉFLEXIONS DE BASE}

Avant d'aborder le cœur du sujet, il est utile de rappeler quelques constatations de base, qui ont étayé la réflexion.

La population se concentre de plus en plus dans les villes aux dépens des zones rurales. Par rapport à la population, elle-même en forte croissance, la part des citadins est ainsi passée de $16 \%$ en 1900 à $45 \%$ en 1990, avec plus de $60 \%$ prévu à horizon 2025. On constate également une multiplication du nombre des villes millionnaires : 157 en 1975, 320 en 2000. 
On estime qu'une grande partie des habitants n'ayant accès ni à l'eau potable, ni à l'assainissement ( 1,4 milliard et 2,9 milliards d'individus respectivement) vit en milieu urbain défavorisé.

Dans les quartiers défavorisés, l'impossibilité technique (absence de voirie, même à l'état de trace), institutionnelle (occupation souvent illégale du sol) et financière (coût d'une desserte classique par réseaux/branchements par rapport à la capacité de payer) de fournir à court terme un service conventionnel en eau et assainissement aux populations concernées constitue un problème considérable.

Face à l'explosion urbaine et à l'accroissement incontrôlé des populations périurbaines, " l'eau du robinet à domicile pour tous " ne peut pas constituer un objectif en soi : la généralisation des branchements particuliers n'est pas concevable à court terme, si l'on considère l'insuffisante maturité du tissu urbain. De la même façon, le " tout à l'égout " ne peut être considéré comme la solution immédiate et sa mise en place nécessite une période de transition.

Ainsi, les institutions internationales parlent de " niveaux de service ", “ d'accès à l'eau potable ", " d'amélioration des conditions de vie ". Ces termes n'impliquent pas nécessairement l'approvisionnement en eau et l'assainissement selon les standards occidentaux et il est maintenant admis que l'on puisse offrir des services différenciés.

L'approche " technicienne " qui misait sur un service universel indifférencié doit être remplacé par une approche "client" offrant des solutions adaptées.

Une approche client suppose d'abord de bien connaître les populations à desservir. Or, les populations des secteurs défavorisés ne constituent pas un groupe homogène, loin de là.

Plutôt que d'essayer d'établir une typologie des quartiers, le rapport présente des facteurs, par grandes catégories : ceux pouvant avoir un impact principal sur le montage institutionnel, ceux qui révèlent une acceptabilité plus ou moins facile ou un refus des populations et ceux qui déterminent surtout des choix techniques adaptés.

Barrios humildes, villas miserias, favelas, zonas invadidas, slums, kampung, bidonvilles, pueblos jovenes, shanty town, etc., ces diverses appellations recouvrent des réalités bien différentes. Avant toute chose, il faut savoir quels types de services leur sont offerts, et à quel coût parce que, d'une manière ou d'une autre, les populations ont toujours un accès à l'eau.

\section{III — LA PROBLÉMATIQUE}

La desserte des quartiers défavorisés pose des problèmes très spécifiques qui doivent être pris en compte au tout début d'un contrat, à savoir :

- coûts de raccordements trop élevés,

- coûts de gestion de la clientèle trop élevés dus à :

- un taux élevé de factures impayées,
- un fort taux de consommations non facturées ou frauduleuses,

- un faible niveau de consommation individuelle et une fréquence de facturation élevée,

- un nombre élevé d'interventions d'entretien.

A ces contraintes s'ajoutent des particularités dans certaines zones d'habitats spontanés :

- bien souvent, les autorités ne souhaitent pas " légaliser " l'implantation, donc évitent toute reconnaissance officielle que procurent les services publics,

- la sécurité des personnes et des biens y est mal assurée rendant difficiles les opérations d'entretien et de recouvrement,

- le fichier client est pratiquement impossible à établir,

- le mode de facturation est mal adapté,

- tout est en situation précaire : les habitations, les populations, les consommations.

\section{IV — LE POINT DE VUE DE L'USAGER}

Pour les habitants des grands centres urbains, se procurer de l'eau potable constitue une nécessité vitale, qui se transforme souvent en préoccupation quotidienne pour les populations défavorisées éloignées des points d'eau.

Pour les populations n'ayant pas accès au réseau, les problèmes de disponibilité de l'eau et de coût du service se traduisent par une recherche constante d'une juste consommation : l'objectif de l'usager consiste ainsi à concilier son besoin vital en eau avec sa capacité budgétaire, tout en minimisant la pénibilité de la " corvée d'eau ". Cette stratégie le conduit souvent à adopter des modes d'approvisionnement complémentaires, adaptés aux exigences de qualité des différents usages de l'eau. De cette façon, il choisira préférentiellement :

— d'exploiter les ressources " gratuites " (puits privés, mares, rivières, etc.) sans limitation pour les usages non alimentaires, dans la mesure où ces ressources sont à proximité de l'habitation,

- d'acheter chaque jour la quantité d'eau potable nécessaire à l'alimentation (" alternatives " : livreur à domicile, camion-citerne, borne-fontaine, revente de voisinage, etc.), en la consommant de manière parcimonieuse pour limiter les frais.

Mais les ressources dites " gratuites" ne le sont pas, elles sont même très chères si on tient compte de tous les coûts (temps, matériel, etc.). Quant aux ressources dites " alternatives ", elles sont aussi beaucoup plus chères (jusqu'à 30 fois le prix du mètre cube livré au réseau). Paradoxalement, ce sont donc les pauvres qui paient l'eau le plus cher, si l'on se réfère au prix unitaire du mètre cube d'eau potable réellement utilisé.

Des modes d'approvisionnement complémentaires sont aussi utilisés par les populations raccordées au réseau, lorsque le service est intermittent.

La faiblesse et les habitudes budgétaires conduisent les consommateurs à payer comptant de petites ou très 
petites sommes pour des volumes d'eau limités. La culture de la facture périodique a posteriori leur est souvent suspecte, et leur apparaît impossible à gérer.

Des systèmes de prépaiement existent et sont mis en œuvre surtout en Afrique du Sud, soit pour la livraison d'eau du réseau vers des réservoirs individuels, soit en utilisant des compteurs à prépaiement.

Le niveau de satisfaction de l'usager conditionne presque toujours sa volonté de payer : ainsi, on constate que dans les quartiers défavorisés, les ménages à faible revenu sont prêts à payer dans la mesure où le service fourni répond à un réel besoin.

\section{ALTERNATIVES COMMERCIALES}

L'objectif du fournisseur consiste à offrir un service de proximité qui réponde aux attentes de l'usager final (qualité des prestations, modalités de paiement), tout en étant économiquement rentable, et compatible avec les souhaits du concédant.

Aujourd'hui, les grands centres urbains des pays émergents voient coexister deux types de fournisseurs offrant des prestations complémentaires :

- le secteur institutionnel représenté par le Service des Eaux,

- le secteur non institutionnel constitué de livreurs d'eau, de revendeurs au micro-détail, de revente de voisinage, de vidangeurs de fosses septiques, - et dont l'existence, sinon légale, peut être tolérée, voire encouragée.

Parfois complémentaires pour une même clientèle, ces deux systèmes entretiennent des rapports plus ou moins conflictuels : leurs rôles respectifs dans la vie économique évoluent au cours du temps, selon leur capacité à répondre aux besoins des consommateurs, et à s'adapter dans un environnement concurrentiel.

Plusieurs mécanismes financiers sont présentés dans le rapport et visent à alléger les coûts supportés par les populations défavorisées : péréquation, tranche sociale, modalités de paiement, participation de la main-d'œuvre aux travaux, subventions, branchements sociaux, fonds de travaux, microprêts, prépaiement, tontines, etc.

\section{D ALTERNATIVES TECHNIQUES}

La conception des premières installations d'approvisionnement en eau des habitats spontanés et des zones urbaines défavorisées doit répondre à un certain nombre de critères techniques.

- L'eau fournie doit être potable et conforme aux normes établies par les autorités locales.

- L'approvisionnement initial doit porter sur 20 litres par personne et par jour $(1 / \mathrm{p} / \mathrm{j})$. La conception doit tenir compte de l'hypothèse d'une augmentation de la demande pouvant atteindre environ $150 \mathrm{l} / \mathrm{p} / \mathrm{j}$ à mesure que le niveau de vie s'améliore.

- Des niveaux de service différents peuvent être envisagés.
- Le système de distribution d'eau doit prendre en compte le développement futur.

- Les coûts d'installation, de maintenance et d'exploitation doivent être réduits au minimum sur toute la durée de vie de la concession.

Les différents types de desserte envisagés concernent : les puits communaux à ciel ouvert, les forages, les vendeurs d'eau, les camions-citernes publics, les kiosques à eau, les bornes-fontaines communales, les réservoirs dans la cour ou sur le toit, les robinets dans la cour, et finalement les branchements à domicile.

L'expérience de Durban illustre bien le concept de solutions transitoires pour les quartiers défavorisés. Trois alternatives de desserte en eau sont proposées :

- le réseau pleine pression, qui correspond au réseau public conventionnel,

- le réseau semie pression, qui consiste à remplir un réservoir individuel de 200 litres situé sur le toit de l'habitation, à partir du réseau principal relié au réservoir par des canalisations de faible diamètre,

- un système des réservoirs individuels, dont le principe est le suivant : il s'agit d'installer à côté de chaque foyer un réservoir de 200 litres, relié à un collecteur muni d'un robinet d'arrêt, lui-même rattaché au réseau principal. Une fois par jour, la vanne est ouverte par un régisseur nommé par les services publics, et les réservoirs sont remplis. La consommation est ainsi limitée à 200 litres/foyer/jour.

Dès lors que nous sommes en mesure de fournir plus de 40 litres d'eau potable par personne et par jour, il est nécessaire d'offrir à la population des solutions efficaces pour l'assainissement.

Les solutions transitoires présentées au rapport concernent : les latrines, les systèmes d'assainissement à godets, les latrines ventilées améliorées (VIP), les WC avec traitement sur place, les WC avec traitement des effluents décantés, les fosses septiques, et finalement les réseaux, conventionnels ou simplifiés.

\section{VII $\square$ AMORCE D'UNE APPROCHE SUEZ LYONNAISE DES EAUX}

Le rapport propose une approche permettant d'appréhender au mieux la situation des quartiers défavorisés. Cette approche se résume ainsi :

- développer des contrats appropriés,

- intégrer la problématique des quartiers défavorisés dans le plan directeur, le plus en amont possible,

- procéder à la mise en œuvre de solutions transitoires et évolutives en :

- démocratisant les processus de décision,

- s'appuyant sur l'action de médiateurs reconnus,

- intégrant et encadrant les secteurs non institutionnels.

Les recherches sont poursuivies sur un certain nombre de problèmes clés identifiés au cours de cette première démarche. 
Les discussions du groupe de travail ont fait ressortir un point capital : on ne peut pas aborder la question des quartiers défavorisés de la manière traditionnelle. On doit innover aussi bien dans l'approche du problème, que dans la mise en œuvre de solutions. Cela constitue en soi une révolution culturelle que nous devons faire à l'intérieur du Groupe. Dans la ligne des travaux initiés par Suez Lyonnaise des Eaux, la Banque Mondiale, dans le cadre de son projet de partenariat dénommé " Business Partners for Development ", a constitué avec divers ONG et des grandes sociétés de service d'eau et d'assainissement un groupe permanent d'études et de recherche sur ce thème. A cette occasion, Suez Lyonnaise des Eaux a publié un ouvrage intitulé " Solutions alternatives pour l'approvisionnement en eau et l'assainissement des quartiers défavorisés " sur l'ensemble de cette recherche, à laquelle a été attribué un Trophée de l'Innovation (Prix spécial du Jury) pour 1998. 